\section{Die Gründung der Arsi-Universität}

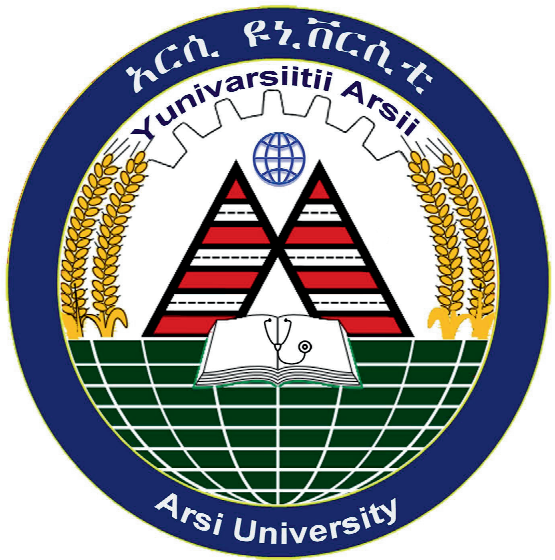

Das Logo der Arsi University

Ende 2014 formierte sich in Asella auf Betreiben der äthiopischen Regierung im Rahmen des Programms zur Etablierung neuer Stätten zur höheren Aus- und Weiterbildung in allen Teilen des Landes aus den bisher der Universität von Adama zugehörigen Fakultäten

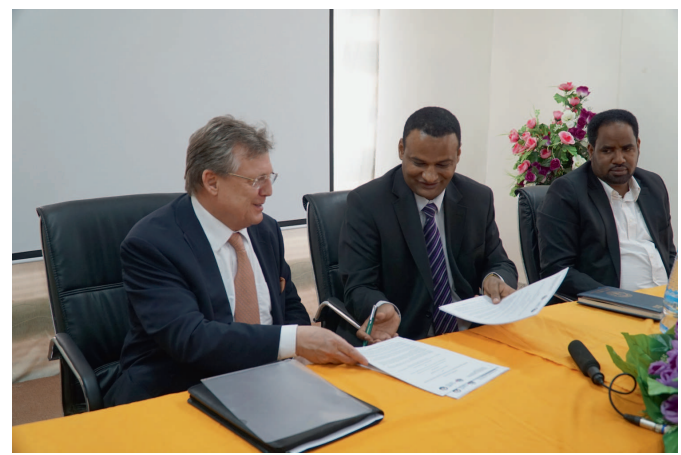

Vertragsunterzeichnung im April 2015, v. I. n. r.: Professor Dr. Dieter Häussinger (Direktor des HITM), Dr. Tolla Beriso (ehem. Präsident der Arsi University), Dr. Hirpho Teno (ehem. Vizepräsident der Arsi University)

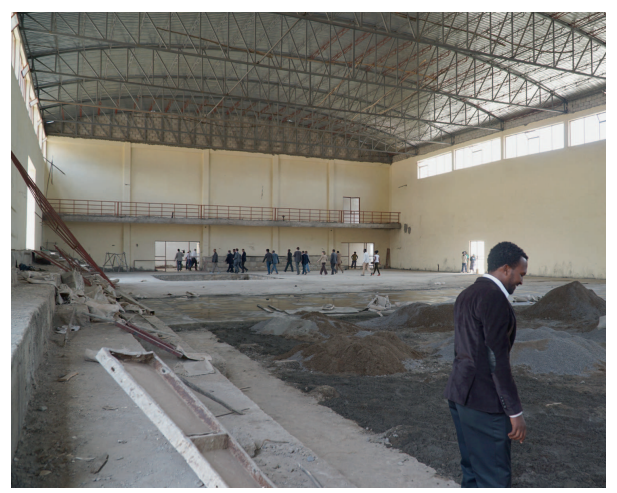

Die im Bau befindliche Sporthalle der Arsi University

(Medizin, Rechtswissenschaften, Agrarwissenschaften, Geisteswissenschaften, Wirtschaftswissenschaften und der Sportakademie) die neu gegründete Arsi University. Als neue Universität der Arsi-Zone mit Hauptsitz in Asella übernahm sie die Leitung der Medizinischen Fakultät und damit auch die lokale Verantwortung für die Partnerschaft mit der HeinrichHeine-Universität. Der neue Präsident der Arsi University, Dr. Beriso, bekräftigte von Beginn an sein großes Interesse, die Partnerschaft unverändert fortzuführen und weiterentwickeln zu wollen. Diese Absichtserklärung wurde im Rahmen eines Besuchs von Professor Dr. Häussinger mit einer deutschen Delegation in Asella im April 2015 durch Unterzeichnung einer entsprechenden vertraglichen Kooperationserklärung niedergelegt. Mit der Arsi University wurde in Äthiopien ein Partner gewonnen, der nach einer Internationalisierung der angebotenen Aus- und Weiterbildung strebt und an einer Akademisierung der Ausbildung interessiert ist. Anlässlich der Neugründung hat kürzlich auch ein dringend benötigter Aus- und teilweiser Neubau des Krankenhauses in Asella begonnen. 


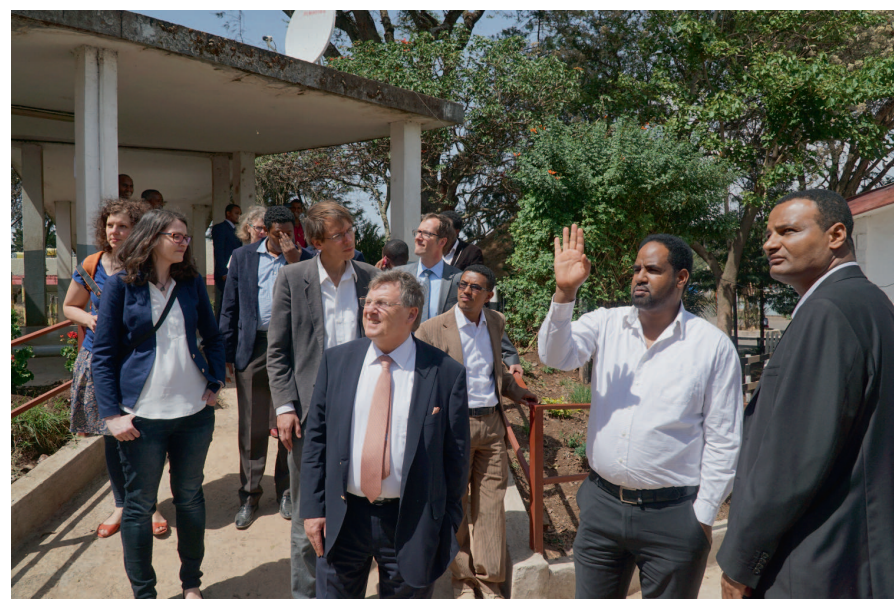

Rundgang über das Krankenhausgelände beim Treffen der deutschen und äthiopischen Delegationen (April 2015)

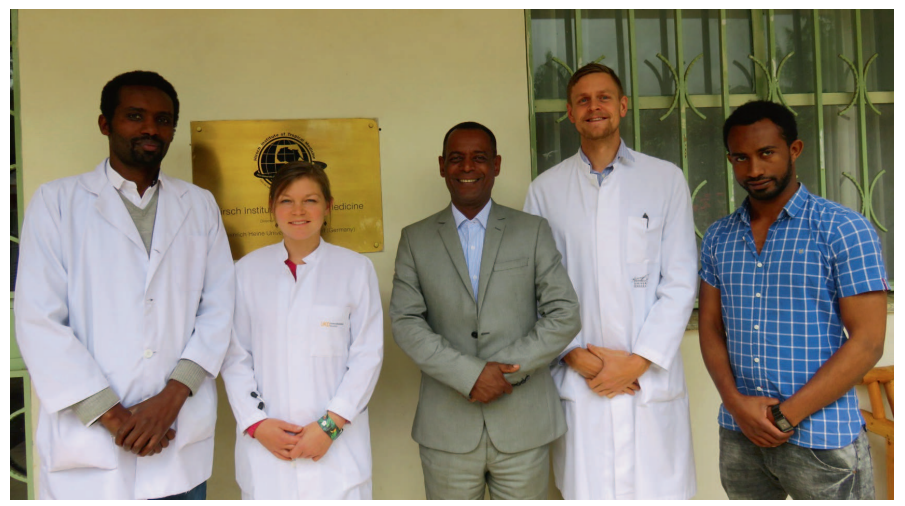

Ein Treffen mit Dr. Duguma, dem neuen Präsidenten der Arsi University

In den Folgejahren nach der Universitätsgründung wurde Dr. Tolla Beriso zum Bildungsminister des Bundesstaates Oromia befördert, und Dr. Duguma übernahm die Position des Universitätspräsidenten. Unter Dr. Dugumas Leitung zeigt sich die Arsi-Universität weiterhin als verlässlicher und enthusiastischer Partner in der wissenschaftlichen und administrativen Zusammenarbeit. 


\section{Arsi, Land der Athleten}

Die Liste der olympischen Medaillengewinner aus Äthiopien ist beeindruckend. Bisher konnten die Sportler aus Äthiopien 53 Medaillen, alle bei den Sommerspielen, erringen. Besonders herausragend sind die international bekannten Marathonläufer, wie beispielsweise Haile Gebrselassie, der 1973 in Asella geboren wurde. Er stellte insgesamt 26 Weltrekorde auf, war zweifacher Olympiasieger (1996 Atlanta und 2000 Sydney: Gold, 10.000-Meter-Lauf) und ist viermaliger Gewinner des Berlin-Marathons sowie dreifacher Sieger des hochdotierten Dubai-Marathons.

In Asella fand im April 2016 die feierliche Eröffnung des Tirunesh Dibaba Athletics Training Center statt, welches nach einer äthiopischen Spitzenathletin benannt wurde. Das Trainingscenter beherbergt auf 45 Hektar Fläche nicht nur eine 400-Meter-Aschenbahn, sondern unter anderem auch ein Fußballfeld, Tennisplätze, Aufenthaltsräume für Athleten und Trainer, eine Bibliothek, eine Klinik und ein Verwaltungsgebäude.

Kenenisa Bekele ist einer der weltbesten Langstreckenläufer. Auch er stammt aus dem Land Oromia. Er ist nicht nur dreifacher Olympiasieger, sondern hält auch derzeit die Weltrekorde über 5.000- und 10.000-Meter-Langstrecke (Stand: September 2016). Auch Spitzenathletinnen stammen aus der Region: Derartu Tulu konnte über die 10.000Meter-Strecke zweimal die olympische Goldmedaille erringen, 1992 in Barcelona und 2000 in Sydney. Frau Tulu ist Inhaberin des ersten Hauses am Platze in Asella. Sie führt das nach ihr benannte Deratu Tulu Hotel, welches in verschiedenen Internetportalen sehr gelobt und empfohlen wird. Das dortige sehr gute Restaurant hat die Mitarbeiter des Instituts schon häufiger zum Verweilen eingeladen.

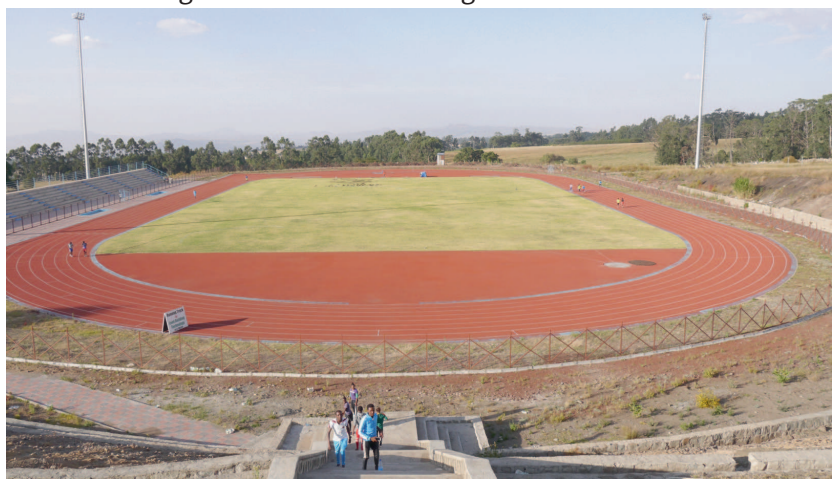

Aschenbahn des Tirunesh Dibaba Athletics Training Centers der Sportakademie der Arsi-Universität in Asella 


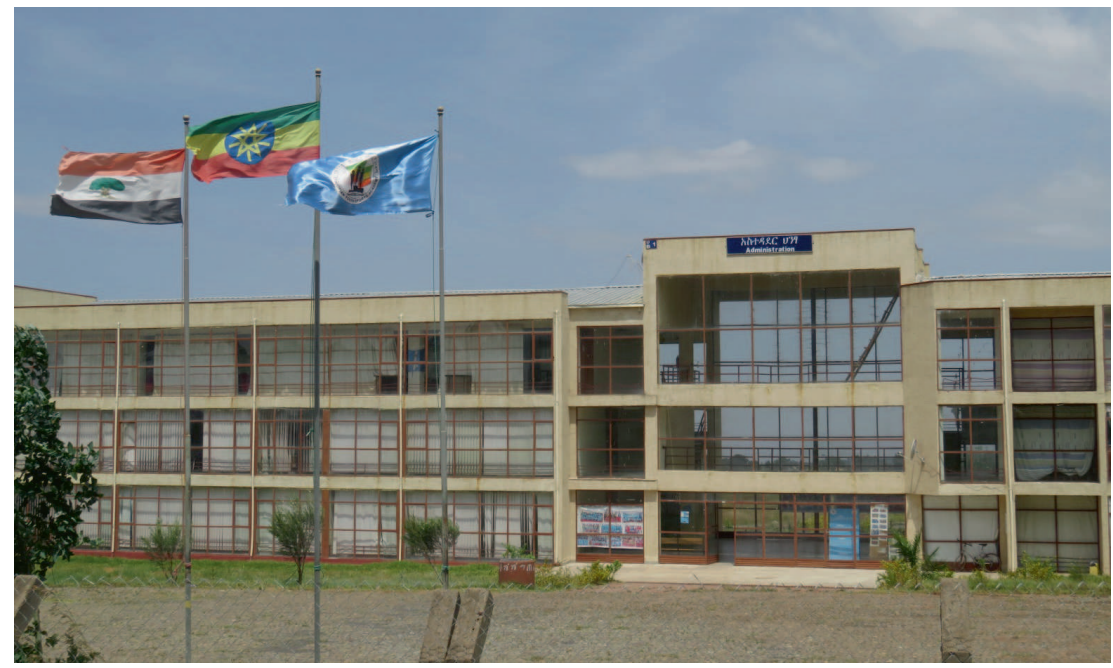

Der Campus des Tirunesh Dibaba Training Centers

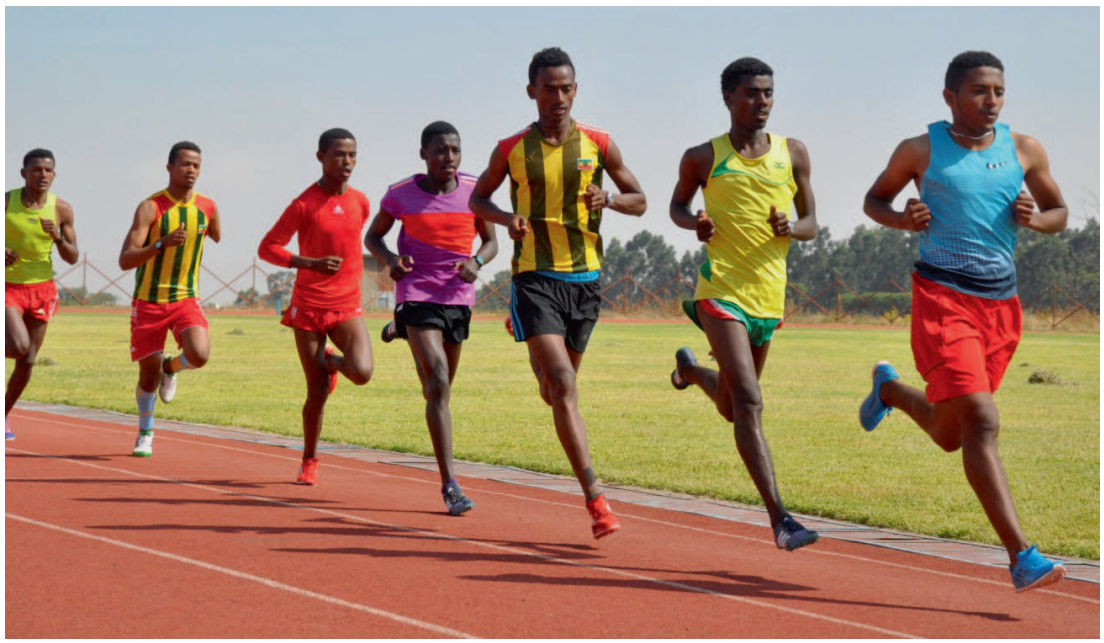

November 2016: Junge Athleten beim Training 
Diese äthiopischen Läufer trainieren unter einfachen Bedingungen in der Umgebung der Sportschule und damit auch in unmittelbarer Nähe unserer Wohnhäuser. So kommt es, dass die Koordinatoren des Instituts jeden Morgen auf dem Weg zur Arbeit dem internationalen Spitzensport begegnen.

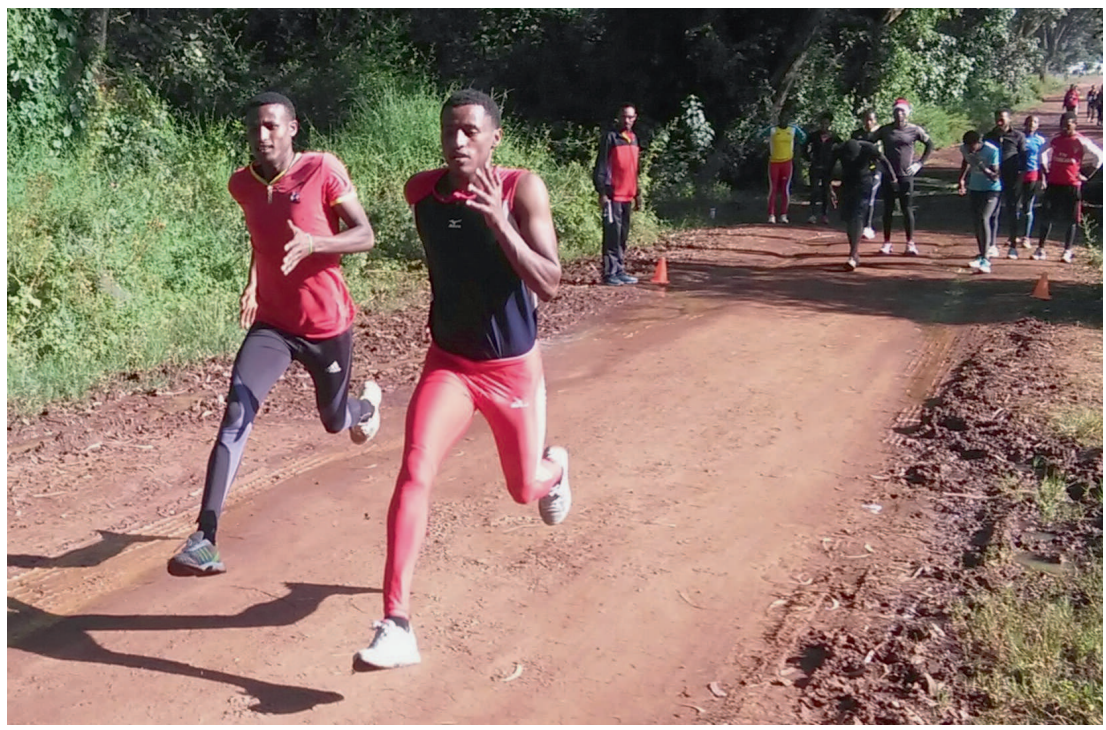

Professionelles Lauftraining auch unter einfachen Bedingungen: Nicht das gesamte Training kann im neuen Athletics Training Center stattfinden 


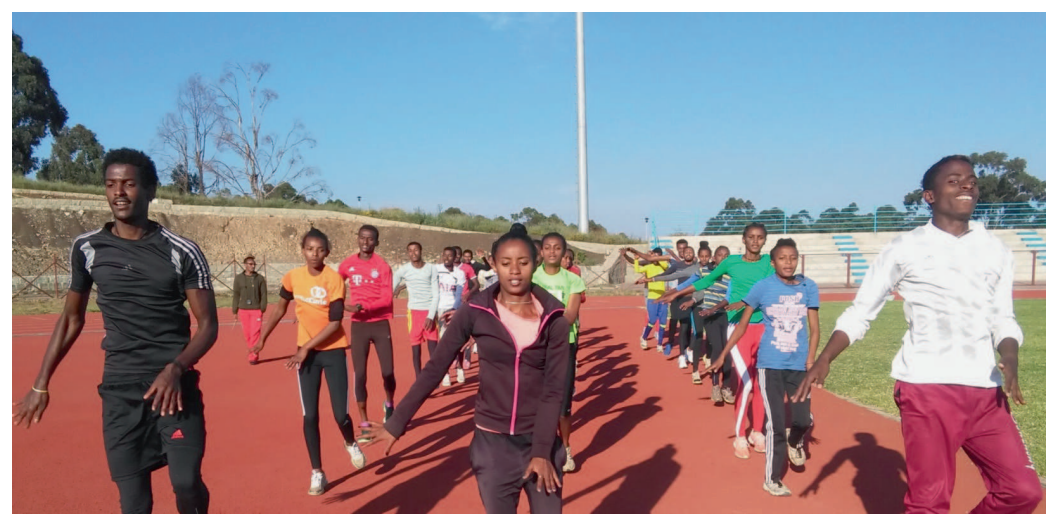

Trainingseinheiten

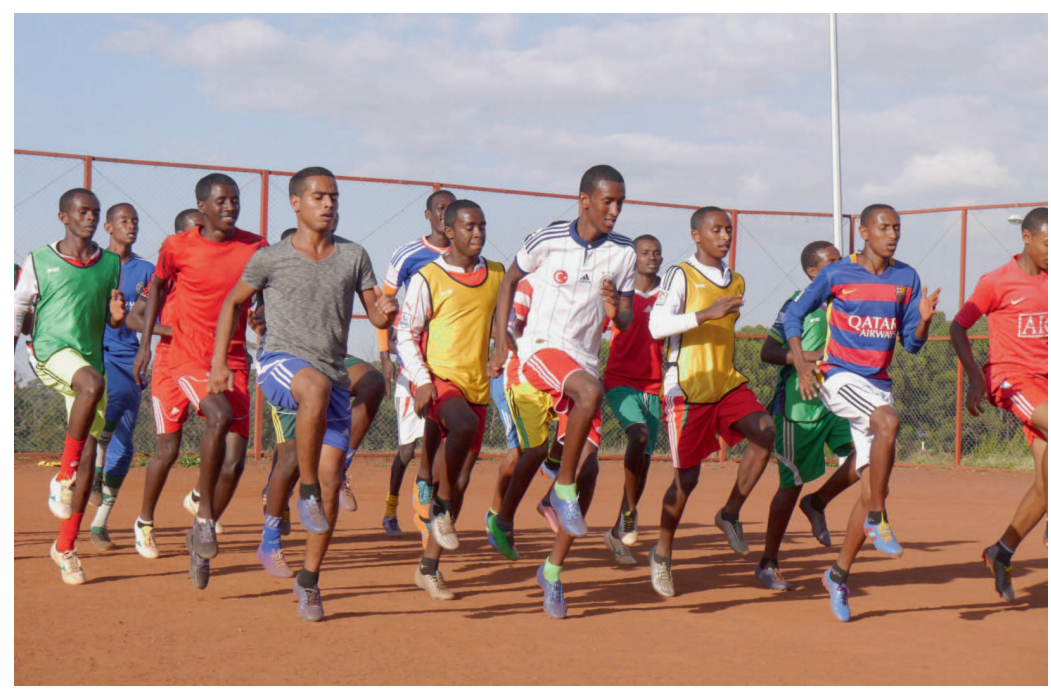

Junge Läufer aus Arsi 